\title{
Optical detection of paramagnetic defects in diamond using off-resonance excitation of NV centers
}

\author{
Nir Alfasi, Sergei Masis, Oleg Shtempluck, and Eyal Buks \\ Andrew and Erna Viterbi Department of Electrical Engineering, Technion, Haifa 32000 Israel
}

(Dated: April 8, 2019)

\begin{abstract}
In this work we use fluorescence from nitrogen-vacancy defects in diamond to detect and explore other paramagnetic defects in the diamond, such as P1 defects, which are commonly undetectable through optical detection of magnetic resonance in standard conditions. Our method does not require overlap between the defects' resonances and therefore is applicable in a wide region of magnetic fields and frequencies, as verified by excellent fit to theoretical predictions. We propose a depolarization scheme of P1 defects to account for the observed data. To verify our results, we perform cavity-based detection of magnetic resonance and find a good agreement between the measured optically induced polarization and the value obtained theoretically from rate equations. The findings in this work may open the way to detection of paramagnetic defects outside of the diamond through the photoluminesence of nitrogen-vacancy defects, which might be useful for imaging in biology.
\end{abstract}

PACS numbers: 76.30.Mi, 81.05.ug, 42.50.Pq

\section{INTRODUCTION}

The nitrogen-vacancy (NV) defect in diamond consists of a substitutional nitrogen atom $(\mathrm{N})$ combined with a neighbor vacancy (V) [1]. In its negatively-charged state the $\mathrm{NV}^{-}$defect has a spin triplet ground state [2] having relatively long coherence time [3]. The $\mathrm{NV}^{-}$spin state can be polarized via the process of optically-induced spin polarization (OISP) [4, 5] and can be measured using the technique of optical detection of magnetic resonance (ODMR) [6 8]. These properties facilitate a variety of applications including magnetometry [9-16] and sensing [3, 10, 17, 18].

ODMR of diamond samples having relatively low density of spin defects is commonly described using a singlespin model, in which dipolar coupling between different spins is disregarded. However, this approach becomes invalid when the spin density is sufficiently high. In general, the strength of the dipolar coupling can be characterized by the magnetic susceptibility $\chi$. At the Larmor frequency, the magnetic susceptibility of a spin $1 / 2$ ensemble having a number density $n_{\mathrm{S}}$ is given by $\chi=i\left(n_{\mathrm{S}} / n_{\mathrm{S} 0}\right) P_{z 0}$, where $P_{z 0}$ is the spin polarization in steady state and the density $n_{\mathrm{S} 0}$ is given by $n_{\mathrm{S} 0}=$ $4 T_{2}^{-1} / \hbar \gamma_{\mathrm{e}}^{2} \mu_{0}$, where $T_{2}^{-1}$ is the transverse spin relaxation rate, $\gamma_{\mathrm{e}}=2 \pi \times 28.03 \mathrm{GHz} \mathrm{T}^{-1}$ is the electron spin gyromagnetic ratio and $\mu_{0}$ is the permeability of free space [19]. The transverse spin relaxation rate of our sample $T_{2}^{-1} \simeq 0.1 \mathrm{MHz}$ yields the value of $n_{\mathrm{S} 0} \simeq 10^{17} \mathrm{~cm}^{-3}$, which is about 3 times smaller than the number density of $\mathrm{NV}^{-}$defects in our sample. Thus, when the polarization $\left|P_{z 0}\right|$ becomes of order unity by applying OISP [4, 5], $\chi$ cannot be treated as a small parameter, and dipolar coupling cannot be disregarded.

In the current study we investigate diamond samples having relatively high density of both $\mathrm{NV}^{-}$and nitrogen 14 substitution (P1) defects. The spin density of our samples is not sufficiently high to allow access to the region where macroscopic magnetic ordering occurs [20], however it is sufficiently high to make $\chi \gtrsim 1$, i.e. to make effects originating from dipolar coupling detectable. To further enhance such effects, the sample is cooled down to a cryogenic temperature of $3.6 \mathrm{~K}$ in order to decrease the rate of thermal polarization, which in turn makes OISP more efficient.

While only transitions between spin states with magnetic quantum numbers $m_{\mathrm{s}}= \pm 1$ and $m_{\mathrm{s}}=0$ of $\mathrm{NV}^{-}$ are commonly visible in the ODMR spectrum of diamond samples, our ODMR measurements reveal magnetic resonances of P1 defects [21 23], as well as resonances due to carbon $13(\mathrm{C} 13)$ atoms, $\mathrm{NV}^{-}+1 \rightarrow-1$ transitions and a resonance of unknown origin around $2 \mathrm{GHz}$ found at magnetic fields near the $\mathrm{NV}^{-}$ground-state level anticrossing (GSLAC). The observation of nuclear and electronic spin transitions in $\mathrm{P} 1$ defects can be attributed to driving-induced depolarization and a cross-relaxation process [24 26] between the $\mathrm{NV}^{-}$defects and the target spins under driving. These processes might be of interest in the context of nuclear spins ensembles hyperpolarization [27 29], with $\mathrm{NV}^{-}$defects providing an optical path for readout and control.

To further explore dipolar coupling in our samples, the method of cavity-based detection of magnetic resonance (CDMR) is employed [30]. As discussed below, the results of the CDMR measurements provide further insight into the underlying mechanism responsible for dipolar coupling in our samples. In particular, these results clearly rule out the possibility that heating plays an important role in this mechanism.

In addition, the CDMR measurements allow studying the process of OISP in general, and the prospects of employing OISP for the generation of spin population inversion, which, in-turn, may allow the realization of a diamond-based maser. A maser is constructed by coupling an ensemble of emitters to a microwave cavity and by applying external pumping for inducing population inversion. A maser has a variety of applications, including amplification of microwave signals, high-precision tim- 
ing, optical to microwave conversion, magnetometry and spectroscopy. The process of OISP can be used for inducing population inversion in diamond when the externally applied magnetic field is tuned to the region above the $\mathrm{NV}^{-}$GSLAC point. This together with the relatively long coherence time [3] and long energy relaxation time [31], makes the $\mathrm{NV}^{-}$defects in diamond useful for the construction of a maser, as has been recently proposed [32] and demonstrated experimentally [33]. In this work, we generate population inversion, though we do not reach the masing threshold due to anomalous saturation of the polarization of $\mathrm{NV}^{-}$spins, which is consistent with previous observations [26, 34].

\section{EXPERIMENTAL SETUP}

The experimental setup is schematically depicted in Fig. 1. Defects in a [110] type Ib diamond are created using $2.8 \mathrm{MeV}$ electron irradiation with a dose of approximately $8 \times 10^{18} \mathrm{e} / \mathrm{cm}^{2}$, followed by annealing at $900^{\circ} \mathrm{C}$ for 2 hours and acid cleaning, resulting in the formation of $\mathrm{NV}^{-}$defects with density of $n_{\mathrm{S}} \simeq 3.25 \times 10^{17} \mathrm{~cm}^{-3}$ [44].

Two multimode optical fibers are glued to the irradiated diamond using optical adhesive. The one labeled as F1 in Fig. 1(a) is used for delivering laser light having wavelength of $532 \mathrm{~nm}$ to the sample, and the one labeled as F2 transmits the photoluminescence (PL) emit-
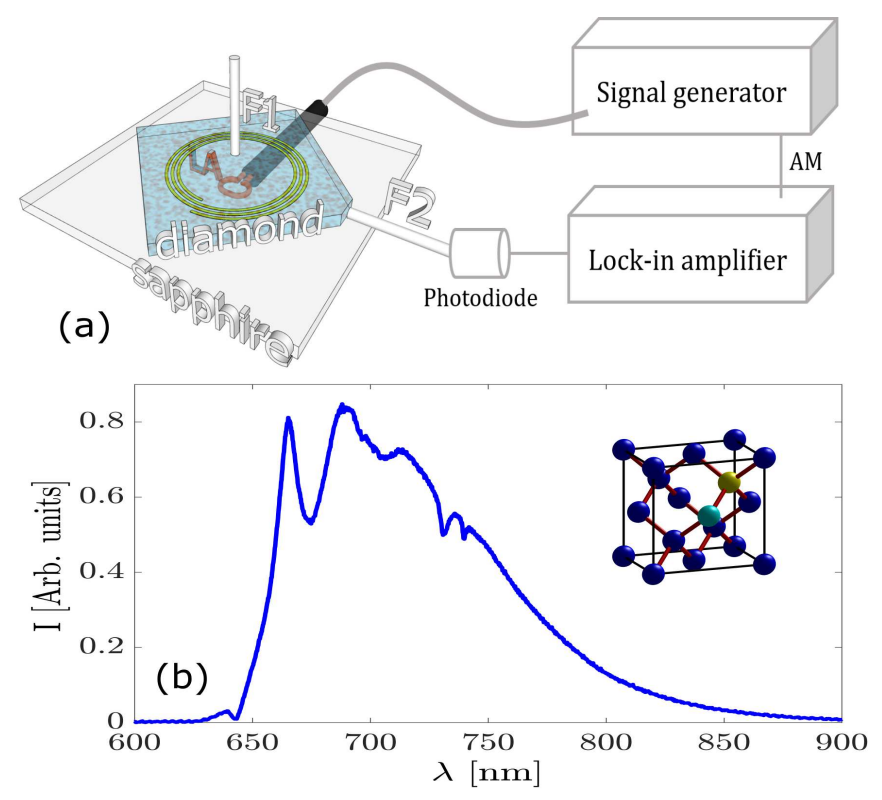

FIG. 1: Experimental setup. (a) Two multimode optical fibers F1 and F2 are glued to the diamond, which is attached to a sapphire wafer supporting a spiral-shaped superconducting resonator. A loop antenna (LA) transmits both input and output microwave signals. (b) PL intensity $I$ as a function of optical wavelength $\lambda$. ted from the sample. The diamond is attached to a sapphire wafer supporting a spiral shaped superconducting microwave cavity (resonator) [45, 46]. The spiral is made of $180 \mathrm{~nm}$ thick niobium, has 7-turns, inner diameter of $0.7 \mathrm{~mm}$ and width and spacing of $40 \mu \mathrm{m}$. In the first part of this work, the delivered light intensity is relatively high and consequently the resonator is heated above its critical temperature and is effectively disabled, while in the second part, the light intensity is reduced and the resonator may be used as a superconducting cavity. A three-loop antenna (LA) is placed in the vicinity of the diamond. An amplitude modulated signal at frequency $f_{\mathrm{p}}$ is injected into the LA, and the modulation signal serves as a reference for a lock-in amplifier connected to a photodiode collecting the filtered PL $(605-700 \mathrm{~nm})$. A superconducting solenoid magnet is used for applying magnetic field $\mathbf{B}$. The setup is cooled down to a base temperature of $3.6 \mathrm{~K}$ to enhance hyperpolarization of non-NV impurities [47].

The spatial orientation of the sample with respect to the externally applied magnetic field is characterized by a unit vector $\hat{\mathbf{n}}_{\mathrm{MA}}=$ $\left(\sin \theta_{\mathrm{MA}} \cos \varphi_{\mathrm{MA}}, \sin \theta_{\mathrm{MA}} \sin \varphi_{\mathrm{MA}}, \cos \theta_{\mathrm{MA}}\right)$. In this work, we use several orientations to confirm our theoretical predictions. The angles for the data presented in Fig. 2 and Fig. 3 are $\theta_{\mathrm{MA}}=-4^{\circ}$ and $\varphi_{\mathrm{MA}}=95^{\circ}$, in Fig. 4 $\theta_{\mathrm{MA}}=-12.3^{\circ}$ and $\varphi_{\mathrm{MA}}=95^{\circ}$, in Fig. 5 $\theta_{\mathrm{MA}}=-9.5^{\circ}$ and $\varphi_{\mathrm{MA}}=94.5^{\circ}$, whereas the angles used for the CDMR measurements in Fig. 6] are $\theta_{\mathrm{MA}}=-1.85^{\circ}$ and $\varphi_{\mathrm{MA}}=97^{\circ}$.

\section{III. $\mathrm{NV}^{-}$}

The $\mathrm{NV}^{-}$ground state spin triplet Hamiltonian is given by [36, 37]

$$
\frac{\mathcal{H}_{\mathrm{NV}-}}{\hbar}=D S_{1, z}^{2}-\gamma_{\mathrm{e}} \mathbf{B} \cdot \mathbf{S}_{1}+\frac{E_{\mathrm{NV}^{-}}\left(S_{1,+}^{2}+S_{1,-}^{2}\right)}{2}
$$

where $D$ is the zero field splitting induced by spin-spin interaction, $\mathbf{S}_{1}=\left(S_{1, x}, S_{1, y}, S_{1, z}\right)$ is a vector electronic spin 1 operator, $\mathbf{B}$ is an externally applied magnetic field, $E_{\mathrm{NV}}$ - is the strain-induced splitting, the raising $S_{1,+}$ and lowering $S_{1,-}$ operators are defined by $S_{1, \pm}=S_{1, x} \pm i S_{1, y}$ and the $\mathrm{NV}^{-}$axis is parallel to the $z$ axis.

The color-coded plot shown in Fig. 2(a) represents the normalized lock-in signal as a function of the frequency of the applied microwave signal $f_{\mathrm{p}}$ and the externally applied stationary magnetic field $B$. In a single crystal diamond the NV defects have four different possible orientations. The two frequencies per orientation corresponding to the transitions between the spin state with magnetic quantum number 0 and the spin state with magnetic quantum number \pm 1 are calculated by diagonalizing the Hamiltonian (1) [see Fig. 3(b)], and are shown in by the black dashed lines in Fig.2(b). A fitting procedure allows 


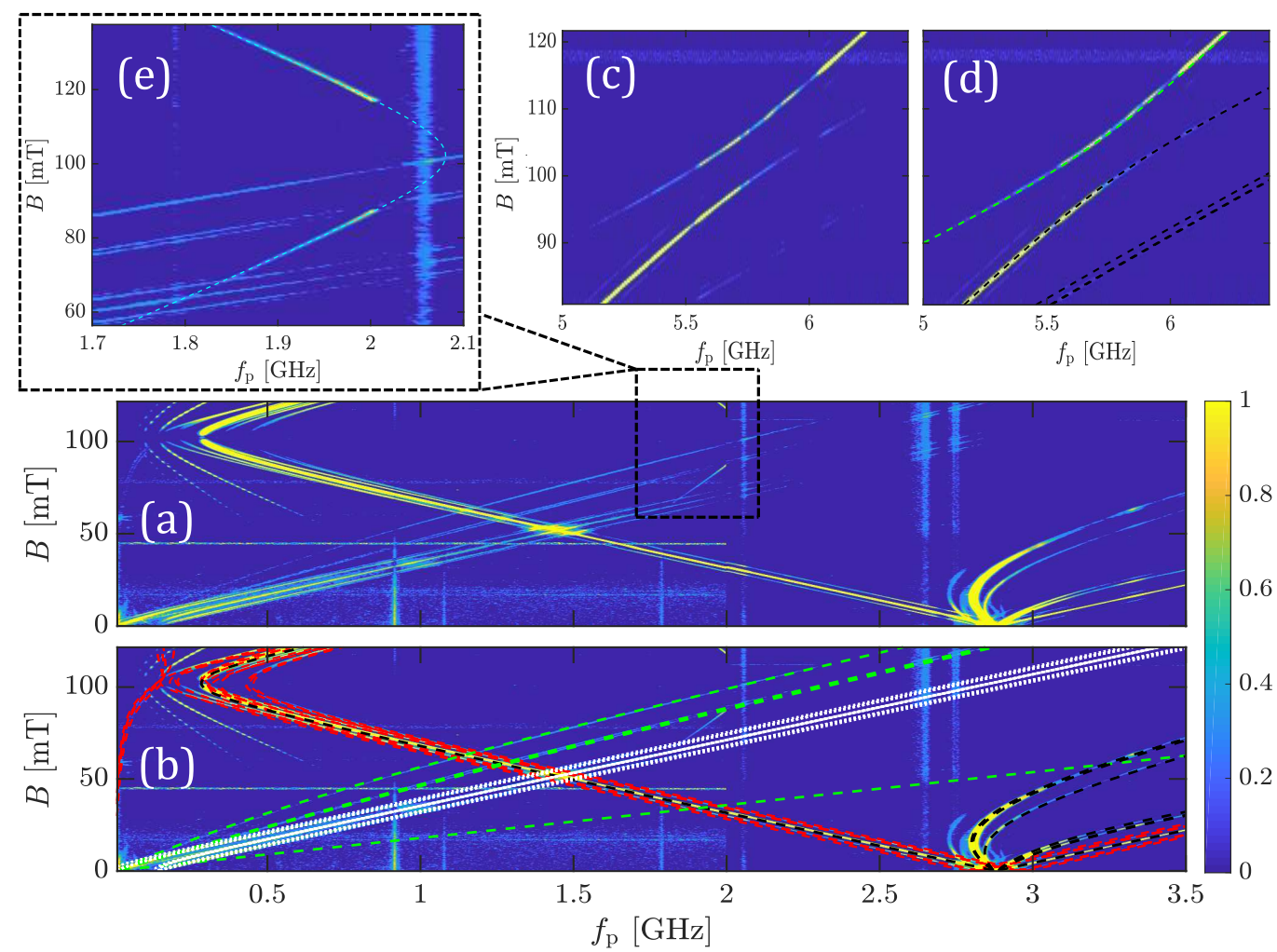

FIG. 2: ODMR. (a) Color coded plot of the normalized ODMR signal as a function of frequency $f_{\mathrm{p}}$ and magnetic field $B$. (b) The overlaid black (green) dashed lines represent $\mathrm{NV}^{-}$ground state \pm 1 to $0(+1$ to -1$)$ transitions and are calculated by numerically diagonalizing the $\mathrm{NV}^{-}$ground state spin triplet Hamiltonian $\mathcal{H}_{\mathrm{NV}}-$ (1). The red dashed lines are obtained by adding the hyperfine interaction term $\mathcal{V}_{\mathrm{NV}^{-}, \mathrm{C}}(2)$ to the Hamiltonian $\mathcal{H}_{\mathrm{NV}^{-}}$(1). The white dotted lines represent P1 electroniclike transitions, calculated using the Hamiltonian $\mathcal{H}_{\mathrm{P} 1}$ (3). Higher oreder hyperbolas corresponding to multi-photon resonances are also observed and are discussed in 35]. The following values are assumed in the calculations: $D=2 \pi \times 2.88 \mathrm{GHz}$, $E_{\mathrm{NV}^{-}}=2 \pi \times 10 \mathrm{MHz}$ [36, 37], $A_{\mathrm{C}, \|}=2 \pi \times 199.7 \mathrm{MHz}, A_{\mathrm{C}, \perp}=2 \pi \times 120.3 \mathrm{MHz}[38-42], \gamma_{\mathrm{n}}=2 \pi \times 3.0766 \mathrm{MHzT}-1$, $Q_{\mathrm{P} 1}=-2 \pi \times 3.97 \mathrm{MHz}, A_{\mathrm{P} 1, \|}=2 \pi \times 114 \mathrm{MHz}$ and $A_{\mathrm{P} 1, \perp}=2 \pi \times 81.3 \mathrm{MHz}$ [43]. (c) ODMR in the high frequency regime showing the ground state level anticrossing (GSLAC). (d) See (b). (e) Resonance lines of unknown origin. Cyan dotted line represents fit to Eq. (4).

determining the sample orientation (characterized by the angles $\theta_{\mathrm{MA}}$ and $\varphi_{\mathrm{MA}}$ ) with respect to the externally applied magnetic field. Additional resonance frequency per $\mathrm{NV}^{-}$orientation is seen in the ODMR spectra and is marked by green dashed lines in Fig. 2(b,d). It corresponds to the transition between states with magnetic quantum numbers +1 and -1 . When the $\mathrm{NV}^{-}$vector and the magnetic field are not strictly parallel, or near the GSLAC, these states acquire a non negligible component of 0 spin state, and hence the transition between them becomes measurable with ODMR [1]. At some combinations of the magnetic field orientation and magnitude, these transitions might be useful for magnetometry with higher responsivity than the one obtained with the more commonly used $0 \rightarrow \pm 1$ transitions, due to the two-times larger pre-factor of $2 \gamma_{\mathrm{e}}$. In order to verify our results, we repeat the same procedure using different orientation of the sample. For both cases excellent fit is obtained (see Figs. 2 and (4).

\section{CARBON 13}

The hyperfine interaction between an $\mathrm{NV}^{-}$defect and the nearest-neighbor carbon- 13 atom has been studied in [38 42]. To account for this interaction, a coupling term $\mathcal{V}_{\mathrm{NV}^{-}, \mathrm{C}}$ given by $[43,48]$

$$
\frac{\mathcal{V}_{\mathrm{NV}^{-}, \mathrm{C}}}{\hbar}=\mathbf{S}_{1} R_{\mathrm{C}}^{-1} \mathcal{A}_{\mathrm{C}} R_{\mathrm{C}} \mathbf{I}_{1 / 2}^{\mathrm{T}},
$$

is added to the Hamiltonian $\mathcal{H}_{\mathrm{NV}^{-}}$(1), where $\mathbf{I}_{1 / 2}$ is a vector nuclear spin $1 / 2$ operator, the matrix $\mathcal{A}_{\mathrm{C}}$ is given by $\mathcal{A}_{\mathrm{C}}=\operatorname{diag}\left(A_{\mathrm{C}, \perp}, A_{\mathrm{C}, \perp}, A_{\mathrm{C}, \|}\right)$, where $A_{\mathrm{C}, \|}$ and $A_{\mathrm{C}, \perp}$ are respectively the longitudinal and transverse hyperfine coupling parameters. The matrix $R_{\mathrm{C}}$ is a rotation matrix satisfying $R_{\mathrm{C}} \hat{\mathbf{n}}_{\mathrm{C}}=\hat{\mathbf{z}}$, where $\hat{\mathbf{n}}_{\mathrm{C}}$ is a unit vector parallel to the vacancy-carbon axis and $\hat{\mathbf{z}}$ is a unit vector in the $z$ direction. The overlaid red dashed lines in Figs. 2(b) and 4(b) are calculated by numerically diagonalizing the $\mathrm{NV}^{-}$Hamiltonian (1) with the added hyperfine coupling term (2). 

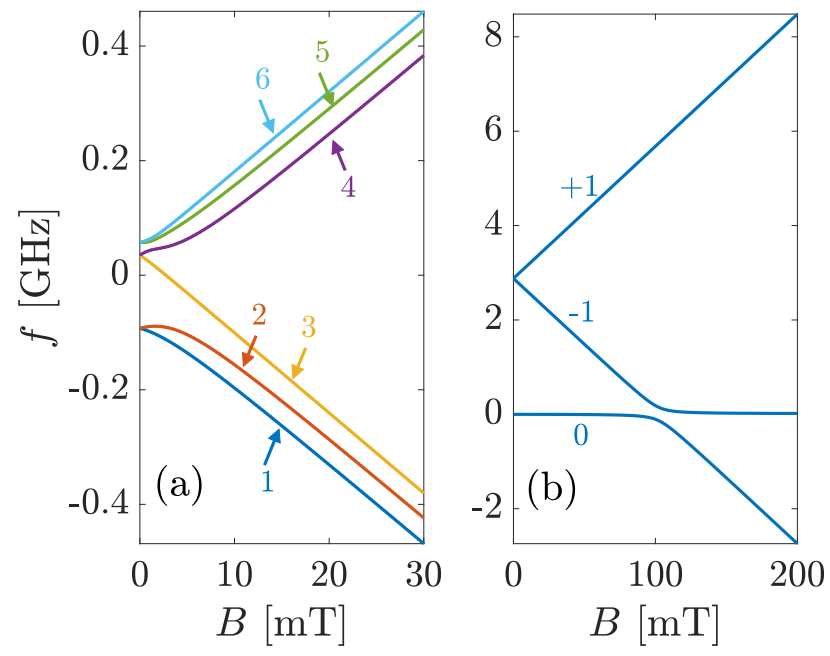

FIG. 3: Energies of P1 (a) [see Eq. (3)] and $\mathrm{NV}^{-}$(b) [see Eq. (1)] states (in frequency units) vs. magnetic field for the case where the angles between the axis and the magnetic field are given by $\theta_{\mathrm{MA}}=-4^{\circ}$ and $\varphi_{\mathrm{MA}}=95^{\circ}$.

\section{P1}

A nitrogen 14 (nuclear spin 1) substitution defect (P1) in diamond [42, 49, 50] has four locally stable configurations. In each configuration a static Jahn-Teller distortion [49] occurs, and an unpaired electron is shared by the nitrogen atom and by one of the four neighboring carbon atoms, which are positioned along one of the lattice directions $\langle 111\rangle[21,27,51,58]$.

The overlaid white dotted lines in Figs. 2(b) and 4(b) are calculated by numerically diagonalizing the $\mathrm{P} 1$ spin Hamiltonian $\mathcal{H}_{\mathrm{P} 1}$ [see Fig. 3(a)], which is given by [43]

$$
\begin{aligned}
\frac{\mathcal{H}_{\mathrm{P} 1}}{\hbar} & =\gamma_{\mathrm{e}} B S_{1 / 2, z}+\gamma_{\mathrm{n}} B I_{1, z}+Q_{\mathrm{P} 1} I_{1, z}^{2} \\
& +\mathbf{S}_{1 / 2} R_{\mathrm{P}}^{-1} \mathcal{A}_{\mathrm{P} 1} R_{\mathrm{P}} \mathbf{I}_{1}^{\mathrm{T}}
\end{aligned}
$$

where $\gamma_{\mathrm{n}}$ is the nitrogen 14 nuclear gyromagnetic ratio, $Q_{\mathrm{P} 1}$ is the nitrogen 14 quadrupole coupling, the matrix $\mathcal{A}_{\mathrm{P} 1}$ is given by $\mathcal{A}_{\mathrm{P} 1}=\operatorname{diag}\left(A_{\mathrm{P} 1, \perp}, A_{\mathrm{P} 1, \perp}, A_{\mathrm{P} 1, \|}\right)$, where $A_{\mathrm{P} 1, \|}$ and $A_{\mathrm{P} 1, \perp}$ are respectively the longitudinal and transverse hyperfine coupling parameters, $\mathbf{S}_{1 / 2}$ is an electronic spin $1 / 2$ vector operator and $\mathbf{I}_{1}$ is a nuclear spin 1 vector operator. The matrix $R_{\mathrm{P}}$ is a rotation matrix satisfying $R_{\mathrm{P}} \hat{\mathbf{n}}_{\mathrm{P}}=\hat{\mathbf{z}}$, where $\hat{\mathbf{n}}_{\mathrm{P}}$ is a unit vector parallel to the $\mathrm{P} 1$ axis.

Higher resolution ODMR measurements of the region of small magnetic field are shown in Fig. 5(a). The overlaid white dotted lines in (b), (c) and (d) represent $\mathrm{P} 1$ resonances. The 6 states per axis orientation are numbered in Fig. 3(a). Electronic-like transitions corresponding to the pairs $(6,1),(5,2)$ and $(4,3)$ are seen in Fig. 5(b), nuclear-like transitions corresponding to the pairs $(6,5),(5,4),(3,2)$ and $(2,1)$ in Fig. 5 (c) and two mixed transitions corresponding to the pairs $(6,3)$ and $(5,3)$ in Fig. 55(d). To our knowledge, this is the first reported observation of P1 nuclear-like transitions through PL of NV defects in diamond.

The visibility of $\mathrm{P} 1$ nuclear and electronic spin transitions in the ODMR data could be explained by resonant microwave heating, however this hypothesis contradicts CDMR measurements, as will be discussed below in Sec. VII Alternatively, we attribute the visibility of $\mathrm{P} 1$ resonances in the ODMR data to a reversehyperpolarization process. In general, large imbalance in spin polarization between $\mathrm{P} 1$ and $\mathrm{NV}^{-}$defects can be induced by applying OISP, which significantly enhances spin polarization of $\mathrm{NV}^{-}$defects. In the absent of any coupling between $\mathrm{P} 1$ and $\mathrm{NV}^{-}$defects, the spin polarization of $\mathrm{P} 1$ defects is expected to be unaffected by the optical pumping, and remains at the value corresponding to thermal equilibrium. However, finite coupling is expected to give rise to optically-induced enhancement in the P1 spin polarization. Such enhancement is observed in our CDMR measurements and is discussed in Sec. VII below. On the other hand, the opposite effect of P1 depolarization can be induced by applying radiofrequency (RF) signal at a frequency close to a P1 transition resonance. Such P1 driving-induced depolarization, in-turn, is expected to give rise to $\mathrm{NV}^{-}$depolarization, due to the coupling between the two ensembles. The observed change in the ODMR signal is attributed according to this mechanism to such an $\mathrm{NV}^{-}$depolarization. Rate equations for this reverse-hyperpolarization process are given in Appendix A.

\section{UNIDENTIFIED RESONANCE}

While we have been able to identify most resonance lines in the ODMR plot, an unidentified pronounced feature appears when better alignment is achieved [see Fig. 2(e)]. The observation that this feature is symmetrical around $\sim 102 \mathrm{mT}$ (GSLAC of $\mathrm{NV}^{-}$) suggests that it originates from a coupling between the $\mathrm{NV}^{-}$defects having axis nearly parallel to $\mathbf{B}$ and a resonance having a magnetic field independent frequency. However, we could not find any evidence for such resonance in our measurements (both optical and microwave reflection). Interestingly, the frequency $F_{\mathrm{f}}$ of this resonance line may be fitted by using the following relation [see cyan dashed line in Fig. 2(e)]

$$
F_{\mathrm{f}}=F_{\mathrm{s}}-\frac{F_{\mathrm{NV}^{-}}}{3}
$$

where $F_{\mathrm{s}}=2.169 \mathrm{GHz}$ and $F_{\mathrm{NV}}$ - is the B-dependent resonance frequency of the $-1 \rightarrow 0$ transition of the $\mathrm{NV}^{-}$defects having axis nearly parallel to $\mathbf{B}$ [see black dashed lines in Fig. 2(b)]. In a different cooling cycle and alignment, we find Eq. (41) to fit the data well with $F_{\mathrm{s}}=2.172 \mathrm{GHz}$. 


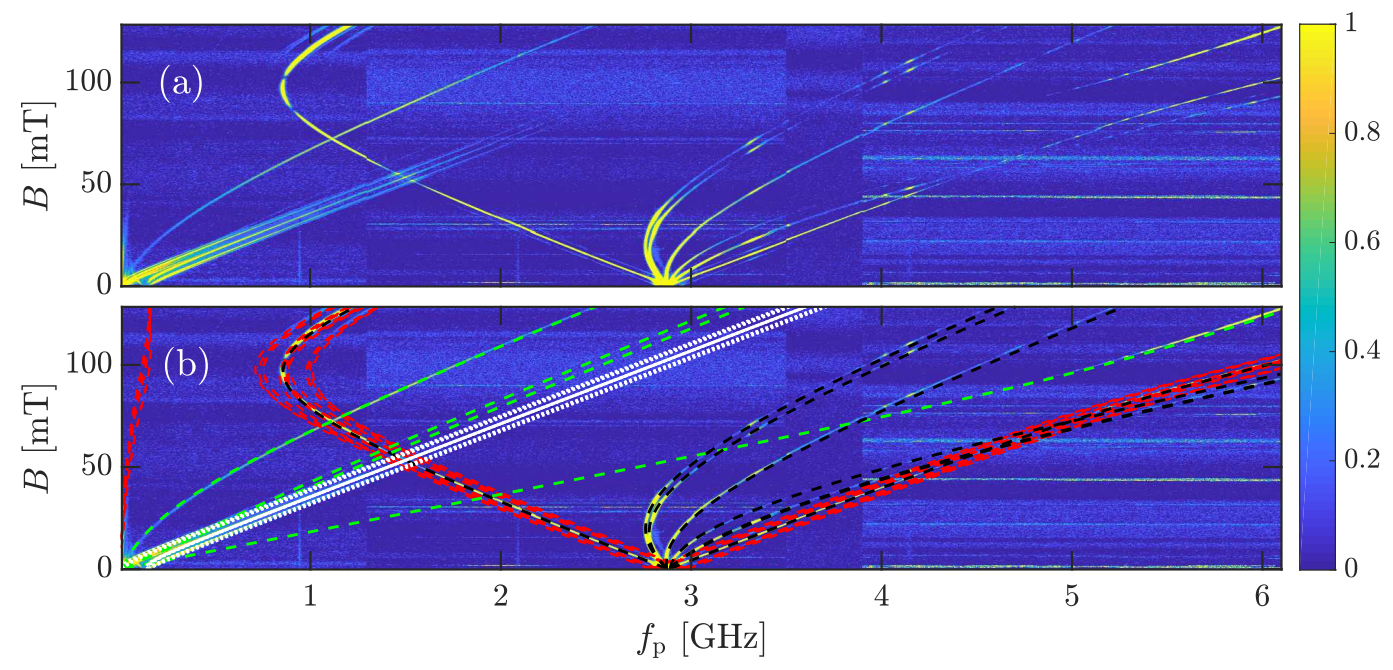

FIG. 4: Rotated configuration. (a) ODMR data obtained by rotating the sample by about $10^{\circ}$ with respect to the configuration used for generating the data shown in Fig. 2(a). (b) Theoretical calculation of resonance lines due to $\mathrm{NV}^{-} \pm 1 \rightarrow 0$ transitions (black), $\mathrm{NV}^{-}+1 \rightarrow-1$ transitions (green), carbon 13 (red) and P1 (white) defects. All lines were obtained by modifying only the diamond rotation angles.

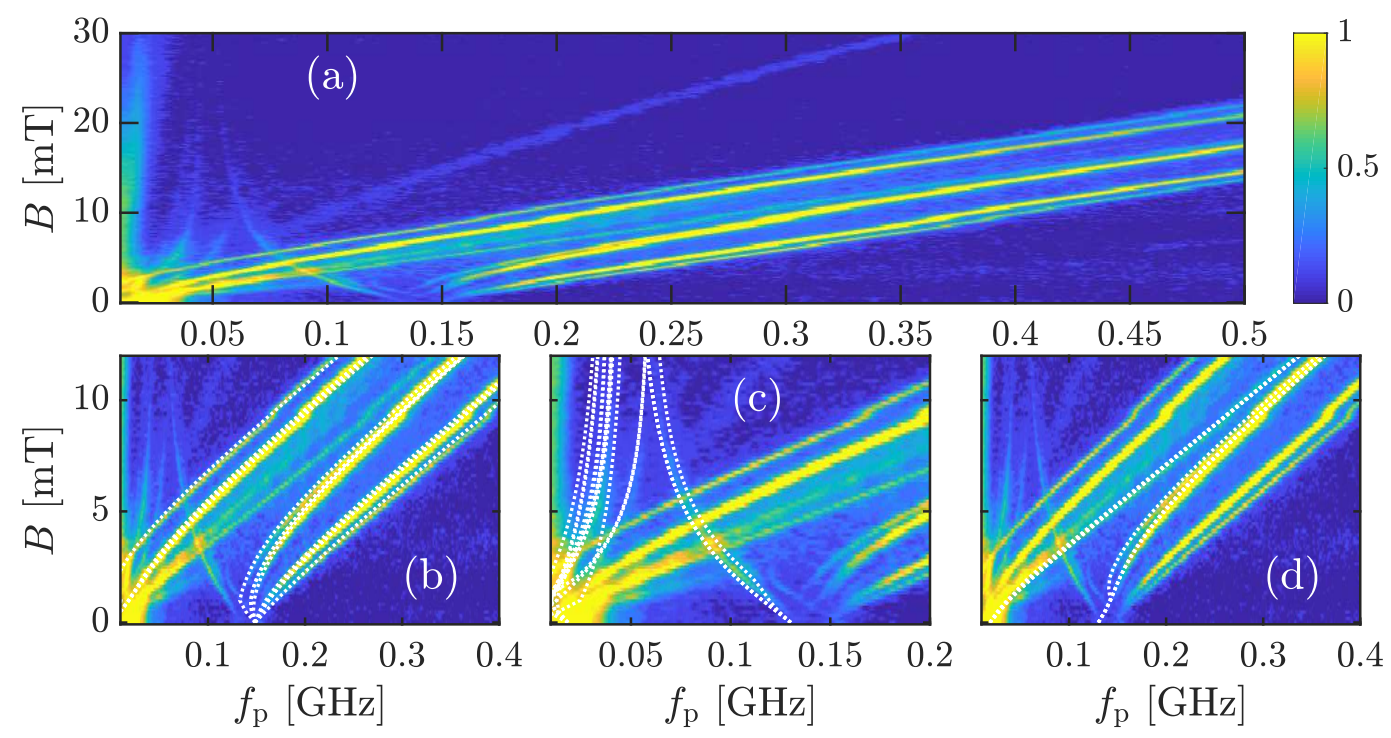

FIG. 5: Normalized ODMR in the region of relatively small magnetic field. The overlaid white dotted lines represent (b) electronic-like, (c) nuclear-like and (d) mixed transitions in P1.

\section{CDMR MEASUREMENTS}

In this section, we reduce the injected light intensity to avoid heating of the superconducting spiral resonator located below our diamond sample, so the resonator regains its superconductive properties and may be used as a cavity. We employ the method of CDMR as discussed in 30]. We apply reflection measurements using a network analyzer to extract the reflection coefficient $S_{11}$, which is given by [59]

$$
S_{11}=\frac{i\left(\omega_{\mathrm{p}}-\omega_{0}\right)-\left(\gamma_{2}-\gamma_{1}\right)}{i\left(\omega_{\mathrm{p}}-\omega_{0}\right)-\left(\gamma_{2}+\gamma_{1}\right)},
$$

where $\omega_{0} \simeq 2 \pi \times 1.464 \mathrm{GHz}$ is the cavity angular resonance frequency, $\omega_{\mathrm{p}}$ is the angular frequency of the injected signal and $\gamma_{1}$ and $\gamma_{2}$ are coupling coefficients associated with the antenna-resonator and the resonatorreservoir (dissipation) couplings, respectively. Note that the cavity resonance frequency is set to the crossing point between $\mathrm{NV}^{-}-1 \rightarrow 0$ and $\mathrm{P} 1$ electronic-like transitions. We fit our data and find $\gamma_{1}=1.15 \mathrm{MHz}$ and 
$\gamma_{2}=0.62 \mathrm{MHz}$ (laser off), leading to a Q-factor of $\sim 2500$, and by assuming $\gamma_{1}$ is independent of laser power, we find $\gamma_{2}$ for various laser powers. Note that $\gamma_{1}>\gamma_{2}$, i.e. the resonator is over-coupled (away from spin resonances). Figure 6(a) shows typical CDMR data with six resonances - five corresponding to P1 transitions and one to the $\mathrm{NV}^{-}-1 \rightarrow 0$ transition, whereas Fig. 6(b) is for magnetic field above the GSLAC, where the ground state of the $\mathrm{NV}^{-}$triplet is the state having $m_{\mathrm{s}}=-1$ and the transition $0 \rightarrow-1$ is seen. In the first case, we expect the coupling between the $\mathrm{NV}^{-}$defects and the superconducting resonator to increase $\gamma_{2}$ due to increased losses. The opposite behavior is expected in the second case, since the state $m_{\mathrm{s}}=0$, which is optically populated via OISP, is no longer the ground state of the system, and consequently some population inversion is expected, resulting in a decrease in $\gamma_{2}$. These phenomena are observed here and are shown in Figs. 6(c)-(d) for various laser intensities $I_{\mathrm{L}}$. For both cases, the process of optically-induced change in $\gamma_{2}$ exhibits a saturation for $I_{\mathrm{L}} \sim 10-15 \mathrm{~mW} \mathrm{~mm}^{-2}$, which is similar to the anomalous saturation reported in [26, 34].

In an attempt to increase the loaded $Q$-factor, we move the antenna further away from the diamond-resonator sample. The reduced coupling to the antenna makes the resonator under-coupled $\left(\gamma_{1}<\gamma_{2}\right)$ with $\gamma_{1}=0.27 \mathrm{MHz}$. We repeat the same measurements and similar results are obtained.

The expected change in normalized cavity damping, which is denoted by $\vartheta=\gamma_{\mathrm{s}} / \gamma_{2}$, where $\gamma_{\mathrm{s}}$ is the spininduced change in the cavity damping rate, can be estimated using Eqs. (4) and (8) of Ref. [30]

$$
\vartheta=\frac{\kappa}{1+\Delta^{2} T_{2}^{2}} \frac{1+\frac{T_{1 \mathrm{O}}^{-1}}{T_{1 \mathrm{~T}}^{-1}} \frac{P_{z \mathrm{SO}}\left(I_{\mathrm{L}}\right)}{P_{z \mathrm{ST}}}}{1+\frac{T_{1 \mathrm{O}}^{-1}}{T_{1 \mathrm{~T}}^{-1}}},
$$

where $\kappa$ is the cooperativity parameter characterizing the coupling between the spins and the cavity mode, $\Delta$ is the frequency detuning between the cavity mode frequency and the spin's transition frequency and $T_{1 \mathrm{O}}^{-1}\left(T_{1 \mathrm{~T}}^{-1}\right)$ is the rate of OISP (thermal relaxation). In steady state and when $T_{1 \mathrm{~T}}^{-1} \gg T_{1 \mathrm{O}}^{-1}$ (i.e. when OISP is negligibly small) the coefficient $P_{z \mathrm{ST}}$ is the value of spin polarization $P_{z}$ in thermal equilibrium. In the opposite limit of $T_{1 \mathrm{O}}^{-1} \gg T_{1 \mathrm{~T}}^{-1}$ (i.e. when thermal relaxation is negligibly small) the coefficient $P_{z \mathrm{SO}}$ is the value of $P_{z}$ in steady state. The rate $T_{1 \mathrm{O}}^{-1}$ of OISP is expressed as $T_{1 \mathrm{O}}^{-1}=C_{\mathrm{O}} \gamma_{\mathrm{O}}$, where $C_{\mathrm{O}}$ is a dimensionless parameter characterizing the absorption efficiency and where $\gamma_{\mathrm{O}}=I_{\mathrm{L}} \sigma \lambda_{\mathrm{L}} / h c$ is the rate of optical absorption, $I_{\mathrm{L}}$ is the laser intensity, $\sigma=3 \times 10^{-17} \mathrm{~cm}^{2}$ [60] is the optical cross section, $h$ is the Plank's constant and $c$ is the speed of light in vacuum. Note that Eq. (6) is obtained by assuming that all contributing spins share the same coupling to the cavity mode and to the laser light and that nonlinearity in the response can be disregarded.

The spin polarization $P_{z}$ as a function of OISP rate $T_{10}^{-1}$ can be extracted from the data shown in Fig. 6] using Eq. (6). Results of the extraction procedure are presented in Fig. 6(e)-(f) using blue pluses (red crosses) markers for $\mathrm{NV}^{-}$(P1) for magnetic fields below [Fig. 6(e)] and above [Fig. 6(f)] the $\mathrm{NV}^{-}$GSLAC. It can be seen from Fig. 6)(e) that when the $\mathrm{NV}^{-}$and $\mathrm{P} 1$ resonances coincide, polarization of both defects is of the same order of magnitude and OISP is $\sim 1.6$ times larger when compared to thermal polarization alone (laser off). The rate equations derived in appendix $\mathrm{A}$ are used to calculate the expected dependency of $P_{z}$ on $T_{1 \mathrm{O}}^{-1}$. Solid lines in Fig. 6(e)-(f) are generated by solving Eqs. (A1) and (A2) (the parameters used for the fit are given in the caption of Fig. 6). The comparison between data and theory yields a good agreement.

In order to gain a further insight, we revisit CDMR data acquired with a different, yet similar, setup as described in [30], where $\mathrm{NV}^{-}$and $\mathrm{P} 1$ resonances do not coincide near the cavity frequency. Same analysis as described above is performed and OISP is found to be about 5 times larger for $\mathrm{NV}^{-}$when compared to thermal polarization alone, but only 1.15 larger for P1 defects. These results suggest that the relative ineffectiveness of OISP that is revealed by Fig. 6) (e) originates from the proximity between the frequencies of $\mathrm{NV}^{-}$and electronic-like P1 transitions.

As was discussed above, when the externally applied magnetic field exceeds the value of $\sim 102 \mathrm{mT}$ (i.e. above the $\mathrm{NV}^{-}$GSLAC) the process of OISP is expected to increase (rather than decrease) the polarization $P_{z}$. The plot in Fig. 6(f) demonstrates this behavior, and shows that this process gives rise to population inversion (i.e. a change in the sign of $P_{z}$ ). The largest measured value of $\left|P_{z}\right|$ is about 15 times larger than the value corresponding to thermal equilibrium. This enhancement factor is significantly larger compared to the case where $B<102 \mathrm{mT}$ [see Fig. 6(e)]. However, it is not sufficiently large to allow reaching the threshold of masing. The threshold inaccessibility occurs due to the above-discussed anomalous saturation in the dependency of polarization upon laser power.

Note that the power of microwave driving used in the CDMR measurements is about 8 orders of magnitude lower than the power used in the ODMR measurements. This fact together with the significant hyperpolarization observed in the CDMR measurements excludes resonant microwave heating effects as being responsible for the visibility of $\mathrm{P} 1$ transitions in the ODMR data presented above.

\section{CONCLUSIONS}

Our results demonstrate that the $\mathrm{P} 1$ defects in diamond and the $\mathrm{NV}^{-}$ground state +1 to -1 transition can be optically probed. In addition to electronic-like transitions in $\mathrm{P} 1$, we report here for the first time on an optical detection of both nuclear-like and mixed transitions. We show that these transitions are visible in a 

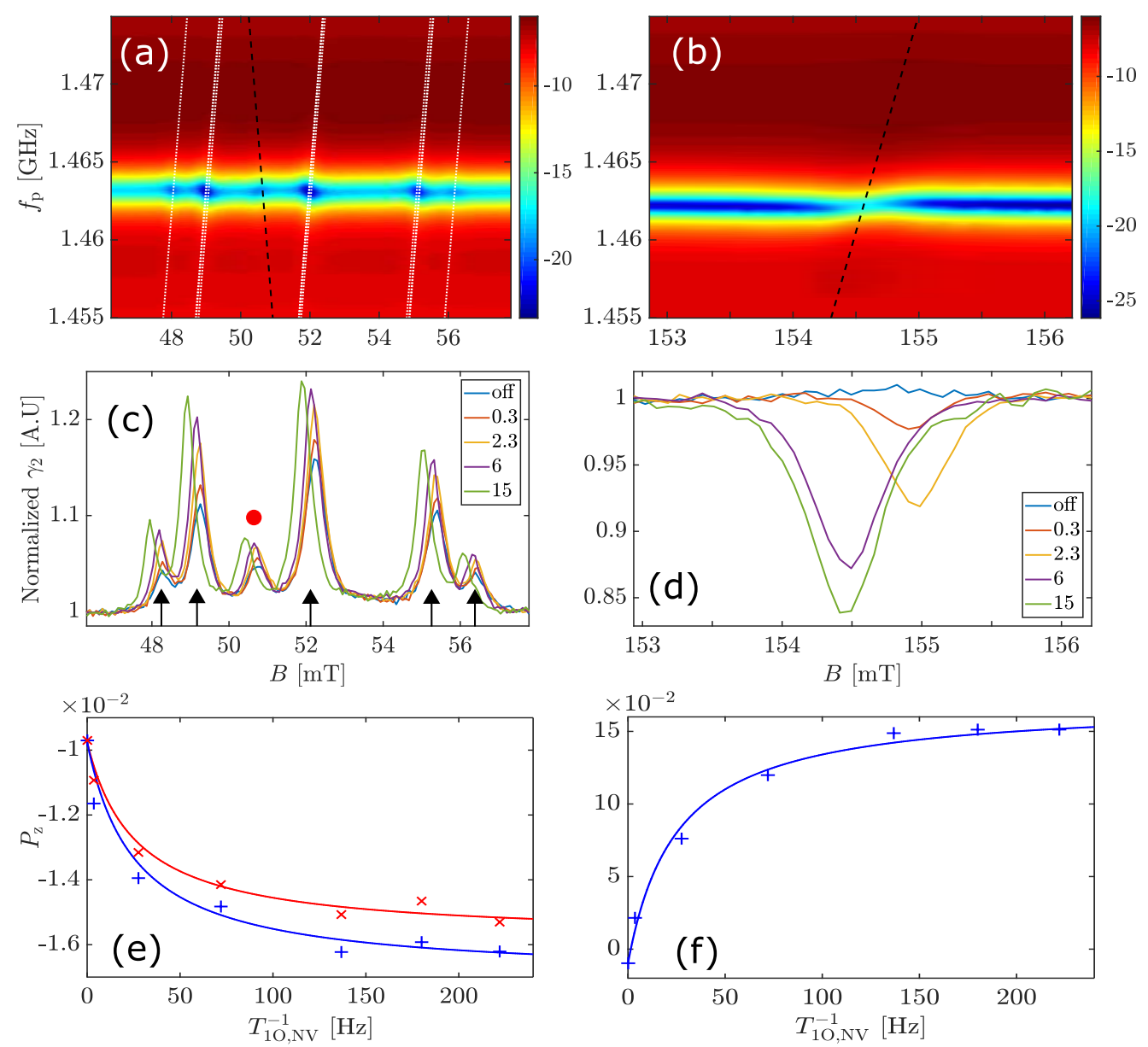

FIG. 6: Cavity mode reflectivity showing (a) five P1 resonances and one $\mathrm{NV}^{-}$resonance and (b) one $\mathrm{NV}^{-}$resonance in the over-coupled resonator case. Fit to theory is shown using black dashed lines for $\mathrm{NV}^{-} 0 \rightarrow-1$ transition [see Eq. (1)] and white dotted lines for P1 defects [see Eq. (3)]. (c)-(d) Change in normalized $\gamma_{2}$ with respect to the magnetic field for various laser powers (legends inside figures indicate values of laser intensity $I_{\mathrm{L}}$ in units of $\mathrm{mW} \mathrm{mm} \mathrm{m}^{-2}$ ). Resonances due to P1 defects are marked by black arrows, while resonance due to $\mathrm{NV}^{-} 0 \rightarrow-1$ transition is marked by a red circle. (e)-(f) Polarization $P_{z}$ as a function of OISP rate $T_{1 \mathrm{O}}^{-1}$. Markers (blue pluses for $\mathrm{NV}^{-}$, red crosses for P1) denote data extracted from (c)-(d), whereas solid lines (blue for $\mathrm{NV}^{-}$, red for P1) represent the steady state numerical solution of the rate equations (A1) and (A2). The parameters used for the calculations are $C_{\mathrm{O}}=1.5, P_{z \mathrm{~T}}=-9.7 \times 10^{-3}, T_{1 \mathrm{~T}, \mathrm{NV}}^{-1}=25 \mathrm{~Hz}, T_{\mathrm{I}, \mathrm{NV}}^{-1}=5 \mathrm{~Hz}, T_{1 \mathrm{~T}, \mathrm{P} 1}^{-1}=8 \mathrm{~Hz}$, $T_{\mathrm{I}, \mathrm{P} 1}^{-1}=40 \mathrm{~Hz}$ and $T_{\mathrm{d}, \mathrm{P} 1}^{-1}=0$.

wide range of frequencies and magnetic field amplitudes. These transitions may enable better sensitivity to magnetic field, which is a key feature in quantum sensing and quantum information. The ability to simultaneously probe the magnetic resonances of both $\mathrm{NV}^{-}$and $\mathrm{P} 1$ can be exploited for sensitivity enhancement by using hyperpolarization of $\mathrm{P} 1$ defects and exploiting the large density of $\mathrm{P} 1$ defects in diamond compared to $\mathrm{NV}^{-}$defects $(\sim 100$ times larger $)$.

In addition, we observe an unknown resonance feature in the ODMR spectrum. This feature is markedly clear and is symmetric around the GSLAC magnetic field, leading us to conclude that it corresponds to coupling between $\mathrm{NV}^{-}$and another unknown resonance. A significant population inversion is observed with $B>102 \mathrm{mT}$.
Further study is needed to understand the mechanism responsible for the anomalous saturation and to overcome it in order to reach the threshold of masing.

\section{ACKNOWLEDGEMENTS}

We greatly appreciate fruitful discussions with $\mathrm{Paz}$ London, Aharon Blank, Efrat Lifshitz, Vladimir Dyakonov, Sergey Tarasenko, Victor Soltamov, Nadav Katz, Michael Stern, Amit Finkler and Nir Bar-Gil. 


\section{Appendix A: Rate equations}

Consider the case where a transverse excitation having amplitude $\omega_{1}$ and angular frequency $\omega_{\mathrm{p}}$ is applied to the $\mathrm{P} 1$ spins having angular transition frequency $\omega_{\mathrm{P} 1}$. In addition OISP is applied to the NV spins having angular transition frequency $\omega_{N V}$. The rate equations for the P1 and NV spin polarizations, which are denoted by $P_{z, \mathrm{P} 1}$ and $P_{z, \mathrm{NV}}$, respectively, are taken to be given by

$$
\begin{aligned}
\frac{\mathrm{d} P_{z, \mathrm{P} 1}}{\mathrm{~d} t} & =-\frac{P_{z, \mathrm{P} 1}-P_{z 0, \mathrm{P} 1}}{T_{\mathrm{P} 1}}, \\
\frac{\mathrm{d} P_{z, \mathrm{NV}}}{\mathrm{d} t} & =-\frac{P_{z, \mathrm{NV}}-P_{z 0, \mathrm{NV}}}{T_{\mathrm{NV}}} .
\end{aligned}
$$

The P1 steady state value $P_{z 0, \mathrm{P} 1}$ is obtained by averaging the contributions of driving-induced depolarization (relevant terms are labeled by the subscript $\mathrm{d}$ ), dipolar coupling to NV spins (subscript I) and thermal relaxation (subscript $\mathrm{T}$ )

$$
P_{z 0, \mathrm{P} 1}=\frac{T_{\mathrm{d}, \mathrm{P} 1}^{-1} \times 0+T_{\mathrm{I}, \mathrm{P} 1}^{-1} P_{z \mathrm{I}, \mathrm{P} 1}+T_{\mathrm{T}, \mathrm{P} 1}^{-1} P_{z \mathrm{~T}, \mathrm{P} 1}}{T_{\mathrm{P} 1}^{-1}},
$$

where $T_{\mathrm{P} 1}^{-1}=T_{\mathrm{d}, \mathrm{P} 1}^{-1}+T_{\mathrm{I}, \mathrm{P} 1}^{-1}+T_{\mathrm{T}, \mathrm{P} 1}^{-1}$ is the total $\mathrm{P} 1$ rate. For the case where the $\mathrm{P} 1$ transverse relaxation time $T_{2, \mathrm{P} 1}$ is much shorter than the longitudinal relaxation time the driving-induced depolarization rate is given by

$$
T_{\mathrm{d}, \mathrm{P} 1}^{-1}=\frac{\omega_{1}^{2} T_{2, \mathrm{P} 1}}{1+\left(\omega_{\mathrm{p}}-\omega_{\mathrm{P} 1}\right)^{2} T_{2, \mathrm{P} 1}^{2}} .
$$

Similarly, the NV steady state value $P_{z 0, N V}$ is obtained by averaging the contributions of dipolar coupling to P1 spins (subscript I), thermal relaxation (subscript $\mathrm{T}$ ) and OISP (subscript O)

$P_{z 0, \mathrm{NV}}=\frac{T_{\mathrm{I}, \mathrm{NV}}^{-1} P_{z \mathrm{I}, \mathrm{NV}}+T_{1 \mathrm{~T}, \mathrm{NV}}^{-1} P_{z \mathrm{~T}, \mathrm{NV}}+T_{\mathrm{O}, \mathrm{NV}}^{-1} P_{z \mathrm{O}, \mathrm{NV}}}{T_{\mathrm{NV}}^{-1}}$

where $T_{\mathrm{NV}}^{-1}=T_{\mathrm{I}, \mathrm{NV}}^{-1}+T_{1 \mathrm{~T}, \mathrm{NV}}^{-1}+T_{\mathrm{O}, \mathrm{NV}}^{-1}$ is the total NV rate.

When higher states are disregarded the P1 and NV thermal polarization coefficients are given by $P_{z \mathrm{~T}, \mathrm{P} 1}=$ $-\tanh \left(\omega_{\mathrm{P} 1} / \omega_{\mathrm{T}}\right)$ and $P_{z \mathrm{~T}, \mathrm{NV}}=-\tanh \left(\omega_{\mathrm{NV}} / \omega_{\mathrm{T}}\right)$, respectively, where $\omega_{\mathrm{T}}=2 k_{\mathrm{B}} T / \hbar, \hbar$ is the Planck's h-bar constant and $k_{\mathrm{B}} T$ is the thermal energy, and the polarization coefficients due to dipolar coupling $P_{z \mathrm{I}, \mathrm{P} 1}$ and $P_{z \mathrm{I}, \mathrm{NV}}$ are given by

$$
\begin{aligned}
P_{z \mathrm{I}, \mathrm{P} 1} & =\tanh \left(\frac{\omega_{\mathrm{P} 1}}{\omega_{\mathrm{NV}}} \tanh ^{-1} P_{z, \mathrm{NV}}\right), \\
P_{z \mathrm{I}, \mathrm{NV}} & =\tanh \left(\frac{\omega_{\mathrm{NV}}}{\omega_{\mathrm{P} 1}} \tanh ^{-1} P_{z, \mathrm{P} 1}\right) .
\end{aligned}
$$

[1] M. W. Doherty, N. B. Manson, P. Delaney, F. Jelezko, J. Wrachtrup, and L. C. Hollenberg, "The nitrogenvacancy colour centre in diamond," Physics Reports, vol. 528, no. 1, pp. 1-45, 2013.

[2] M. Doherty, F. Dolde, H. Fedder, F. Jelezko, J. Wrachtrup, N. Manson, and L. Hollenberg, "Theory of the ground-state spin of the nv- center in diamond," Physical Review B, vol. 85, no. 20, p. 205203, 2012.

[3] G. Balasubramanian, P. Neumann, D. Twitchen, M. Markham, R. Kolesov, N. Mizuochi, J. Isoya, J. Achard, J. Beck, J. Tissler et al., "Ultralong spin coherence time in isotopically engineered diamond," Nature materials, vol. 8, no. 5, pp. 383-387, 2009.

[4] L. Robledo, H. Bernien, T. van der Sar, and R. Hanson, "Spin dynamics in the optical cycle of single nitrogenvacancy centres in diamond," New Journal of Physics, vol. 13, no. 2, p. 025013, 2011.

[5] D. Redman, S. Brown, R. Sands, and S. Rand, "Spin dynamics and electronic states of $\mathrm{n}-\mathrm{v}$ centers in diamond by epr and four-wave-mixing spectroscopy," Physical review letters, vol. 67, no. 24, p. 3420, 1991.

[6] C. S. Shin, C. E. Avalos, M. C. Butler, D. R. Trease, S. J. Seltzer, J. P. Mustonen, D. J. Kennedy, V. M. Acosta, D. Budker, A. Pines et al., "Room-temperature operation of a radiofrequency diamond magnetometer near the shot-noise limit," Journal of Applied Physics, vol.
112, no. 12, p. 124519, 2012.

[7] R. Chapman and T. Plakhotnik, "Quantitative luminescence microscopy on nitrogen-vacancy centres in diamond: Saturation effects under pulsed excitation," Chemical Physics Letters, vol. 507, no. 1, pp. 190-194, 2011.

[8] A. Gruber, A. Dräbenstedt, C. Tietz, L. Fleury, J. Wrachtrup, and C. Von Borczyskowski, "Scanning confocal optical microscopy and magnetic resonance on single defect centers," Science, vol. 276, no. 5321, pp. 20122014, 1997.

[9] J. Maze, P. Stanwix, J. Hodges, S. Hong, J. Taylor, P. Cappellaro, L. Jiang, M. G. Dutt, E. Togan, A. Zibrov et al., "Nanoscale magnetic sensing with an individual electronic spin in diamond," Nature, vol. 455, no. 7213, pp. 644-647, 2008.

[10] V. Acosta, E. Bauch, M. Ledbetter, A. Waxman, L.-S. Bouchard, and D. Budker, "Temperature dependence of the nitrogen-vacancy magnetic resonance in diamond," Physical review letters, vol. 104, no. 7, p. 070801, 2010.

[11] G. Balasubramanian, I. Chan, R. Kolesov, M. Al-Hmoud, J. Tisler, C. Shin, C. Kim, A. Wojcik, P. R. Hemmer, A. Krueger et al., "Nanoscale imaging magnetometry with diamond spins under ambient conditions," Nature, vol. 455, no. 7213, pp. 648-651, 2008.

[12] T. Wolf, P. Neumann, K. Nakamura, H. Sumiya, 
T. Ohshima, J. Isoya, and J. Wrachtrup, "Subpicotesla diamond magnetometry," Physical Review X, vol. 5, no. 4, p. 041001, 2015.

[13] H. Mamin, M. Kim, M. Sherwood, C. Rettner, K. Ohno, D. Awschalom, and D. Rugar, "Nanoscale nuclear magnetic resonance with a nitrogen-vacancy spin sensor," Science, vol. 339, no. 6119, pp. 557-560, 2013.

[14] M. Pelliccione, A. Jenkins, P. Ovartchaiyapong, C. Reetz, E. Emmanouilidou, N. Ni, and A. C. B. Jayich, "Scanned probe imaging of nanoscale magnetism at cryogenic temperatures with a single-spin quantum sensor," Nature nanotechnology, vol. 11, no. 8, p. 700, 2016.

[15] L. Rondin, J.-P. Tetienne, S. Rohart, A. Thiaville, T. Hingant, P. Spinicelli, J.-F. Roch, and V. Jacques, "Stray-field imaging of magnetic vortices with a single diamond spin," Nature communications, vol. 4, p. 2279, 2013.

[16] A. Sushkov, I. Lovchinsky, N. Chisholm, R. L. Walsworth, H. Park, and M. D. Lukin, "Magnetic resonance detection of individual proton spins using quantum reporters," Physical review letters, vol. 113, no. 19, p. 197601, 2014.

[17] F. Dolde, H. Fedder, M. W. Doherty, T. Nöbauer, F. Rempp, G. Balasubramanian, T. Wolf, F. Reinhard, L. Hollenberg, F. Jelezko et al., "Electric-field sensing using single diamond spins," Nature Physics, vol. 7, no. 6, pp. 459-463, 2011.

[18] F. Jelezko, T. Gaebel, I. Popa, A. Gruber, and J. Wrachtrup, "Observation of coherent oscillations in a single electron spin," Physical review letters, vol. 92, no. 7, p. 076401, 2004.

[19] A. Abragam, The principles of nuclear magnetism. Oxford university press, 1961, no. 32 .

[20] H. A. El-Ella, "Magnon condensation in a dense nitrogenvacancy spin ensemble," Physical Review B, vol. 99, no. 2, p. 024414, 2019.

[21] H.-J. Wang, C. S. Shin, S. J. Seltzer, C. E. Avalos, A. Pines, and V. S. Bajaj, "Optically detected crossrelaxation spectroscopy of electron spins in diamond," Nature communications, vol. 5, p. 4135, 2014.

[22] L. Hall, P. Kehayias, D. Simpson, A. Jarmola, A. Stacey, D. Budker, and L. Hollenberg, "Detection of nanoscale electron spin resonance spectra demonstrated using nitrogen-vacancy centre probes in diamond," Nature communications, vol. 7, p. 10211, 2016.

[23] C. M. Purser, V. P. Bhallamudi, C. S. Wolfe, H. Yusuf, B. A. McCullian, C. Jayaprakash, M. E. Flatté, and P. C. Hammel, "Optically-detected broadband paramagnetic resonance spectroscopy using nv centers in diamond," arXiv:1802.09635, 2018.

[24] I. Solomon, "Relaxation processes in a system of two spins," Physical Review, vol. 99, no. 2, p. 559, 1955.

[25] C. Belthangady, N. Bar-Gill, L. M. Pham, K. Arai, D. Le Sage, P. Cappellaro, and R. L. Walsworth, "Dressed-state resonant coupling between bright and dark spins in diamond," Physical review letters, vol. 110, no. 15 , p. $157601,2013$.

[26] M. Loretz, H. Takahashi, T. Segawa, J. Boss, and C. Degen, "Optical hyperpolarization of nitrogen donor spins in bulk diamond," Physical Review B, vol. 95, no. 6, p. 064413, 2017.

[27] S. Takahashi, R. Hanson, J. van Tol, M. S. Sherwin, and D. D. Awschalom, "Quenching spin decoherence in diamond through spin bath polarization," Physical review letters, vol. 101, no. 4, p. 047601, 2008.

[28] R. Fischer, C. O. Bretschneider, P. London, D. Budker, D. Gershoni, and L. Frydman, "Bulk nuclear polarization enhanced at room temperature by optical pumping," Physical review letters, vol. 111, no. 5, p. 057601, 2013.

[29] H.-J. Wang, C. S. Shin, C. E. Avalos, S. J. Seltzer, D. Budker, A. Pines, and V. S. Bajaj, "Sensitive magnetic control of ensemble nuclear spin hyperpolarization in diamond," Nature communications, vol. 4, p. 1940, 2013, article number: 1940.

[30] N. Alfasi, S. Masis, R. Winik, D. Farfurnik, O. Shtempluck, N. Bar-Gill, and E. Buks, "Exploring the nonlinear regime of light-matter interaction using electronic spins in diamond," Physical Review A, vol. 97, no. 6, p. 063808 , 2018.

[31] J. Harrison, M. Sellars, and N. Manson, "Measurement of the optically induced spin polarisation of nv centres in diamond," Diamond and related materials, vol. 15 , no. 4, pp. 586-588, 2006.

[32] L. Jin, M. Pfender, N. Aslam, P. Neumann, S. Yang, J. Wrachtrup, and R.-B. Liu, "Proposal for a roomtemperature diamond maser," Nature communications, vol. 6, 2015.

[33] J. D. Breeze, J. Sathian, E. Salvadori, N. M. Alford, and C. W. Kay, "Continuous-wave room-temperature diamond maser," arXiv:1710.07726, 2017.

[34] M. Drake, E. Scott, and J. Reimer, "Influence of magnetic field alignment and defect concentration on nitrogen-vacancy polarization in diamond," New Journal of Physics, vol. 18, no. 1, p. 013011, 2015.

[35] S. Masis, N. Alfasi, R. Levi, O. Shtempluck, and E. Buks, "Multiphoton resonances in nitrogen-vacancy defects in diamond," unpublished, 2019.

[36] P. Ovartchaiyapong, K. W. Lee, B. A. Myers, and A. C. B. Jayich, "Coherent strain-mediated coupling of a single diamond spin to a mechanical resonator," arXiv:1403.4173, 2014.

[37] E. MacQuarrie, T. Gosavi, N. Jungwirth, S. Bhave, and G. Fuchs, "Mechanical spin control of nitrogen-vacancy centers in diamond," Physical review letters, vol. 111, no. 22, p. 227602, 2013.

[38] S. Felton, A. Edmonds, M. Newton, P. Martineau, D. Fisher, D. Twitchen, and J. Baker, "Hyperfine interaction in the ground state of the negatively charged nitrogen vacancy center in diamond," Physical Review $B$, vol. 79, no. 7, p. 075203, 2009.

[39] N. Mizuochi, P. Neumann, F. Rempp, J. Beck, V. Jacques, P. Siyushev, K. Nakamura, D. Twitchen, H. Watanabe, S. Yamasaki et al., "Coherence of single spins coupled to a nuclear spin bath of varying density," Physical review B, vol. 80, no. 4, p. 041201, 2009.

[40] M. Simanovskaia, K. Jensen, A. Jarmola, K. Aulenbacher, N. Manson, and D. Budker, "Sidebands in optically detected magnetic resonance signals of nitrogen vacancy centers in diamond," Physical Review B, vol. 87, no. 22, p. 224106, 2013.

[41] E. Kamp, B. Carvajal, and N. Samarth, "Continuous wave protocol for simultaneous polarization and optical detection of p1-center electron spin resonance," Physical Review B, vol. 97, no. 4, p. 045204, 2018.

[42] J. Loubser and J. van Wyk, "Electron spin resonance in the study of diamond," Reports on Progress in Physics, vol. 41, no. 8, p. 1201, 1978.

[43] A. Cox, M. Newton, and J. Baker, "13c, 14n and 15n 
endor measurements on the single substitutional nitrogen centre (p1) in diamond," Journal of Physics: Condensed Matter, vol. 6, no. 2, p. 551, 1994.

[44] D. Farfurnik, N. Alfasi, S. Masis, Y. Kauffmann, E. Farchi, Y. Romach, Y. Hovav, E. Buks, and N. BarGill, "Enhanced concentrations of nitrogen-vacancy centers in diamond through tem irradiation," Applied Physics Letters, vol. 111, no. 12, p. 123101, 2017.

[45] N. Maleeva, M. Fistul, A. Averkin, A. Karpov, and A. Ustinov, "Electrodynamics of a spiral resonator as a suitable magnetic component of metamaterials," Proceedings of Metamaterials, pp. 474-477, 2013.

[46] N. Maleeva, M. Fistul, A. Karpov, A. Zhuravel, A. Averkin, P. Jung, and A. Ustinov, "Electrodynamics of a ring-shaped spiral resonator," Journal of Applied Physics, vol. 115, no. 6, p. 064910, 2014.

[47] J. H. Ardenkjær-Larsen, B. Fridlund, A. Gram, G. Hansson, L. Hansson, M. H. Lerche, R. Servin, M. Thaning, and K. Golman, "Increase in signal-to-noise ratio of; 10,000 times in liquid-state nmr," Proceedings of the $\mathrm{Na}$ tional Academy of Sciences, vol. 100, no. 18, pp. 10158 $10163,2003$.

[48] C. P. Slichter, Principles of magnetic resonance. Springer Science \& Business Media, 2013, vol. 1.

[49] W. Smith, P. Sorokin, I. Gelles, and G. Lasher, "Electron-spin resonance of nitrogen donors in diamond," Physical Review, vol. 115, no. 6, p. 1546, 1959.

[50] R. Cook and D. Whiffen, "Electron nuclear double resonance study of a nitrogen centre in diamond," Proceedings of the Royal Society of London A: Mathematical, Physical and Engineering Sciences, vol. 295, no. 1441, pp. 99-106, 1966.

[51] R. Hanson, F. Mendoza, R. Epstein, and D. Awschalom, "Polarization and readout of coupled single spins in diamond," Physical review letters, vol. 97, no. 8, p. 087601, 2006.

[52] R. Hanson, V. Dobrovitski, A. Feiguin, O. Gywat, and D. Awschalom, "Coherent dynamics of a single spin interacting with an adjustable spin bath," Science, vol. 320, no. 5874, pp. 352-355, 2008.
[53] D. A. Broadway, J. D. Wood, L. T. Hall, A. Stacey, M. Markham, D. A. Simpson, J.-P. Tetienne, and L. C. Hollenberg, "Spin dynamics of diamond nitrogenvacancy centres at the ground state level anti-crossing and all-optical low frequency magnetic field sensing," arXiv:160\%.04006, 2016.

[54] J. Shim, B. Nowak, I. Niemeyer, J. Zhang, F. Brandao, and D. Suter, "Characterization of hyperfine interaction between single electron and single nuclear spins in diamond assisted by quantum beat from the nuclear spin," arXiv:130\%.025\%, 2013.

[55] B. Smeltzer, L. Childress, and A. Gali, "13c hyperfine interactions in the nitrogen-vacancy centre in diamond," New Journal of Physics, vol. 13, no. 2, p. 025021, 2011.

[56] C. S. Shin, M. C. Butler, H.-J. Wang, C. E. Avalos, S. J. Seltzer, R.-B. Liu, A. Pines, and V. S. Bajaj, "Optically detected nuclear quadrupolar interaction of $n 14$ in nitrogen-vacancy centers in diamond," Physical Review B, vol. 89, no. 20, p. 205202, 2014.

[57] H. Clevenson, E. H. Chen, F. Dolde, C. Teale, D. Englund, and D. Braje, "Diamond-nitrogen-vacancy electronic and nuclear spin-state anticrossings under weak transverse magnetic fields," Phys. Rev. A, vol. 94, p. 021401, Aug 2016.

[58] D. Schuster, A. Sears, E. Ginossar, L. DiCarlo, L. Frunzio, J. Morton, H. Wu, G. Briggs, B. Buckley, D. Awschalom et al., "High-cooperativity coupling of electron-spin ensembles to superconducting cavities," Physical review letters, vol. 105, no. 14, p. 140501, 2010.

[59] B. Abdo, E. Segev, O. Shtempluck, and E. Buks, "Intermodulation gain in nonlinear $\mathrm{NbN}$ superconducting microwave resonators," Appl. Phys. Lett., vol. 88, p. 22508, 2006.

[60] T.-L. Wee, Y.-K. Tzeng, C.-C. Han, H.-C. Chang, W. Fann, J.-H. Hsu, K.-M. Chen, and Y.-C. Yu, "Twophoton excited fluorescence of nitrogen-vacancy centers in proton-irradiated type ib diamond," The Journal of Physical Chemistry A, vol. 111, no. 38, pp. 9379-9386, 2007. 